\title{
Effect of a synthetic pyrethroid deltamethrin on excitability changes following a nerve impulse
}

\author{
PHILIP J PARKIN,* PAMELA M LE QUESNE \\ From the Department of Neurological Studies, The Middlesex Hospital, London and the MRC Toxicology \\ Unit, Carshalton, Surrey, Great Britain
}

SUMMARY Excitability changes following a nerve impulse were studied in the rat tail. Supernormal nerve excitability in control animals was present, as in other vertebrate nerve fibres, from 4-30 ms and was followed by a period of subnormal excitability extending up to 100-200 ms. Two to seven hours after intravenous administration of $1.5 \mathrm{mg} / \mathrm{kg}$ deltamethrin, supernormality was increased in degree and prolonged in duration for up to $400 \mathrm{~ms}$. A minor effect was still detectable 24 hours after injection. This effect of deltamethrin on nerve excitability is probably due to persistent slight depolarisation of the nerve membrane resulting from its known effect of maintaining a proportion of sodium channels open for up to several hundred milliseconds following a nerve impulse.

The pyrethroid insecticides are finding increasing application in pest control as they are extremely potent yet possess low mammalian toxicity. They have been clearly shown to have major electrophysiological effects on the central and peripheral nervous systems of arthropods and vertebrates. Two different clinical syndromes have been described following high doses of pyrethroids to vertebrates. In one, fine tremors occur, and in the other more complex abnormal movements predominate. ${ }^{1}$ These probably reflect differences in the major sites of action of different pyrethroids within the nervous system. Nevertheless the effects of different pyrethroids on nerve membranes and nerve excitability are relatively uniform.

In insect nerve preparations the application of natural pyrethrins causes a marked increase in spontaneous electrical activity resulting in high frequency repetitive discharges, and a train of spikes may be provoked by a single stimulus. At high dosage or following prolonged exposure, conduction block eventually occurs. In vertebrate nerves the effects are similar although repetitive activity appears to be more prominent than the blocking effect. ${ }^{2}$

*Present address: Department of Neurology, North Canterbury Hospital Board, Christchurch, New Zealand.

Address for reprint requests: Dr PM Le Quesne, The Middlesex Hospital Medical School, London W1N 8AA, UK.

Received 19 November 1981

Accepted 9 December 1981
Narahashi and Anderson, ${ }^{3}$ and Narahashi ${ }^{4}$ used the giant axons of the cockroach and later of the squid and crayfish to show that allethrin, one of the earliest synthetic pyrethroids to be developed, induced changes of this kind which were associated with a prolonged negative after potential. At high dosage the action potential gradually decreased in amplitude until conduction block occurred. Using a voltage clamp technique the latter authors correlated these findings with a prolongation in the inward sodium current across the membrane and decrease in the steady state outward potassium current. Similar findings have been described at single nodes of Ranvier following the application of allethrin and more recently of a newer potent synthetic pyrethroid, deltamethrin, previously called decamethrin. ${ }^{56}$ The deltamethrin-induced prolongation of the sodium current appears, however, to be of much longer duration (over one second) than that induced by allethrin (up to one hundred milliseconds). ${ }^{5}$ The long inward sodium current is believed to be due to the prolongation of membrane permeability to sodium resulting from sodium channels which, having opened upon depolarisation, remain open for longer than normal. The effects of pyrethroids on sodium channel kinetics have been the subject of recent study. ${ }^{6} 7$

In normal nerve fibres the sequence of changes in excitability that follow an action potential induced by a single stimulus are now well established and are regarded as a general feature of axonal physiology. ${ }^{8}$ Although much of this evidence is 
derived from isolated nerve preparations, a simple percutaneous technique for demonstrating such changes in the median nerve of humans was described by Gilliat and Willison. ${ }^{9}$ The supernormal and subnormal periods which follow the absolute and relative refractory periods have long been known to correlate with the after potentials that follow a propagated action potential. ${ }^{1011}$ As the after potentials may be modified by alterations in sodium and potassium kinetics across the nerve membrane ${ }^{12}$ we employed a technique in the intact animal similar to that used by Gilliat and Willison in humans, to determine whether the known properties of pyrethroids would be reflected in alterations in the normal sequence of nerve excitability changes following a stimulus.

\section{Methods}

\section{Animals and dosing}

Male Sprague-Dawley rats, ranging in age from 6 to 9 months were used. "Hypnorm" (Janssen) $0.5 \mathrm{ml} / \mathrm{kg}$ intramuscularly was used as the principal anaesthetic and was repeated in smaller doses as necessary. Nitrous oxide and oxygen inhalation was also used in some animals. Deltamethrin (Roussel) was dissolved in glyceralformal $(5 \mathrm{mg} / \mathrm{ml})$. Rats administered deltamethrin received $1.5 \mathrm{mg} / \mathrm{kg}$ via the femoral vein over $5-10$ minutes.

\section{Electrophysiology}

Nerves in the tail were studied using a technique similar to that described by Misumi ${ }^{13}$ who recorded the compound nerve action potential following distal stimulation of the tail when recording proximally. He inferred such action potentials to arise from the sensory fibres because their conduction velocity was greater than for motor nerve fibres calculated from latency differences of the muscle response. Bare steel needle stimulating electrodes inserted distally into the tail were used initially. However, the use of these stimulating electrodes were not always associated with a supernormal response in control animals. Tinned copper wire stimulating electrodes wrapped around the tail were found to give more consistent results and were used in all the experiments reported here. Although care was taken to ensure satisfactory skin contact with electrode paste, an excessively tight application was avoided as the supernormal response may be abolished by nerve ischaemia. ${ }^{9}$ The electrical resistance between the stimulating electrodes was usually 20000 200000 ohms. Two independently variable square wave puses of 50 microvolts duration and up to 200 volts were delivered by a pair of Devices 3072 stimulators and were connected by a diode bridge to an active pair of bipolar electrodes. Bare steel needle electrodes approximately 1-2 $\mathrm{cm}$ apart, were inserted into the proximal end of the tail for recording. Nerve action potentials were recorded using a Medelec MS7 electromyograph and 4-8 sweeps were averaged on a Medelec DAV 7 averager. Potentials were recorded on light sensitive paper using a FOR 7 fibre optic recorder. The recording apparatus was triggered by a Devices Digitimer and triggering could be adjusted so that only the second action potential was recorded. This was particularly convenient with long time intervals between stimuli. The tail was placed on a mat of coiled polythene tubing through which water was pumped from a thermostatically controlled bath. The tail was covered by cotton wool and a perspex box. Tail temperature was monitored by a subcutaneously placed thermistor and was maintained at $31-34^{\circ} \mathrm{C}$.

Two stimuli were used in all experiments. The conditioning stimulus $\mathrm{S} 1$ was adjusted until it was supramaximal. The intensity of the unconditioned stimulus S2 was adjusted to produce a response approximately one third of the amplitude of the response to the supramaximal stimulus. The amplitude of the response to S2 when preceded by $\mathrm{S} 1$ was expressed as a percentage of the amplitude of the response to S2 alone. The initial recording was made at a stimulus interval of $2 \mathrm{~ms}$ and repeated at increasing intervals up to $1000 \mathrm{~ms}$. At each interval the amplitude of $\mathbf{S} 2$ alone was recorded to ensure stability of the preparation. Minor fluctuations in the S2:S1 amplitude ratio did occur at times throughout some experiments and the stimulus intensity of $\mathbf{S} 2$ was therefore adjusted if necessary. Results of an experiment were rejected if $\mathbf{S} 2$ did not remain constant.

In a separate series of experiments the amplitude of the responses to 2.5 or 5.0 volt incremental changes in S2 stimulus were compared to those when preceded by a supramaximal conditioning stimulus at two different stimulus intervals, $8 \mathrm{~ms}$ and $50 \mathrm{~ms}$.

\section{Results}

\section{CONTROL ANIMALS}

The changes in the response to $\mathrm{S} 2$ when preceded by $\mathrm{S} 1$ at varying intervals are illustrated in fig 1 . Twenty two recordings on twenty animals were used to establish the control response. Following the absolute and relative refactory periods, supernormal nerve excitability began at about $4 \mathrm{~ms}$. The wide variation in the responses at $2 \mathrm{~ms}$ and $4 \mathrm{~ms}$ reflects differences in different animals in the duration of the absolute and relative refractory periods. Supernormality reached its maximum at $6-8 \mathrm{~ms}$, and was evident as an increase in the amplitude of the conditioned response compared with that of $\mathbf{S} 2$ alone. At its maximum the mean amplitude of the supernormal response was $129 \%$ of its unconditioned value. Following this peak, supernormality subsequently became less marked and had subsided within $30 \mathrm{~ms}$. There then followed a period of reduced nerve excitability - the subnormal period which persisted until nerve excitability had returned to the unconditioned state at $100-200$ ms stimulus interval.

Eight animals underwent repeated examinations 2-24 hours after the first. Three of them had received glyceralformal in the same amount as administered to the animals receiving deltamethrin $(0.3 \mathrm{ml} / \mathrm{kg})$. 


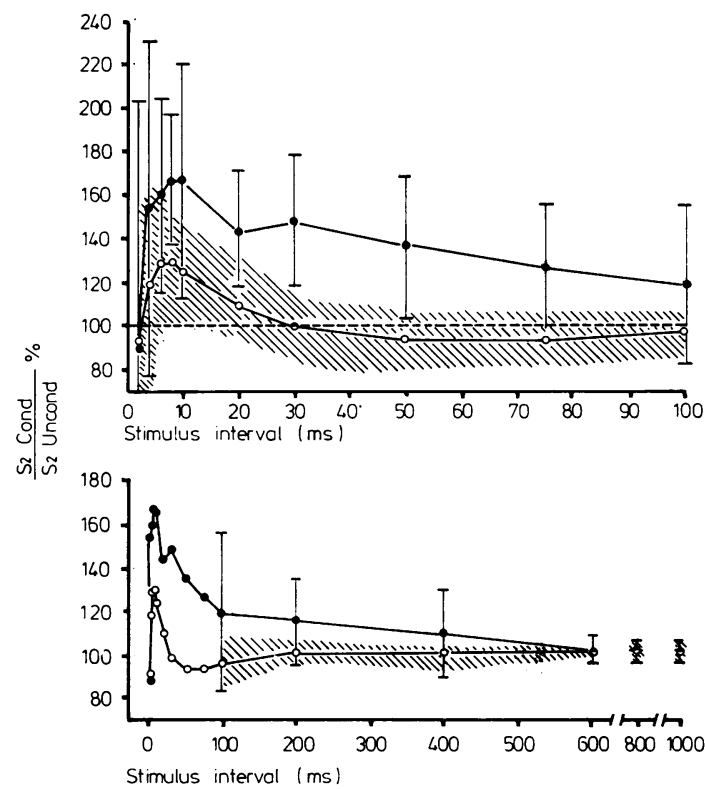

Fig 1 Percentage change in amplitude of response to a submaximal stimulus when preceded by a supramaximal conditioning stimulus at varying intervals. Open circles control animals; closed circles 2-7 hours following deltamethrin. Stippled area denotes $\pm 2 S D$ from the mean in controls; vertical lines $\pm 2 S D$ from the mean in deltamethrin treated animals.

The mean of the results at the first examination and of the second examination showed no significant difference (fig 2).

\section{FOLLOWING DELTAMETHRIN}

Deltamethrin $1.5 \mathrm{mg} / \mathrm{kg}$ was administered intravenously to 17 rats. Five died shortly following injection and before recording could be made. Five recordings were technically unsatisfactory and were rejected. The following observations were made on the seven remaining animals.

Within 10-15 minutes of injection excessive

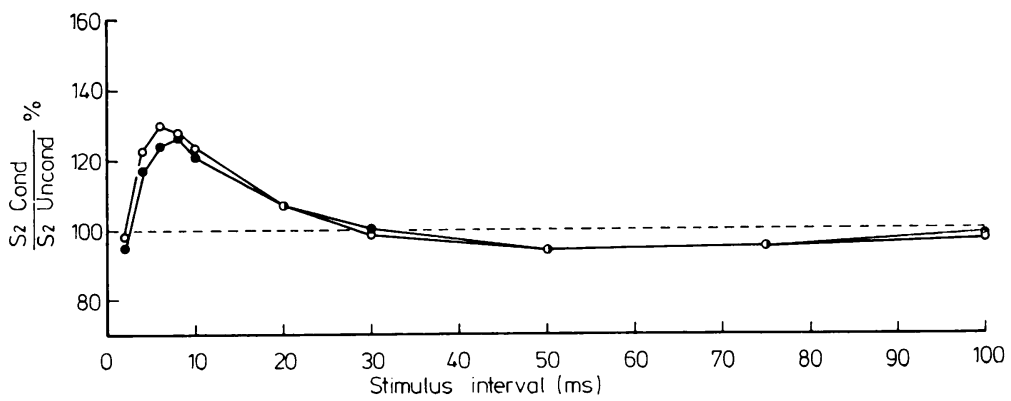

Fig 2 Control animals examined at time 0 (open circles). Repeat examinations 2-24 hours later (closed circles).

salivation occurred and, shortly after, abnormal involuntary movements appeared, often to a marked degree. Twitching movements of the vibrisae usually heralded prominent writhing and jerking movements of the limbs and trunk. Because of the intensity of these movements recording was usually not possible until 2-7 hours after injection by which time the animal was still, although brief jerking movements could often be induced by a loud noise or by sudden movement.

The changes in amplitude of the response to S2 when preceded by $\mathbf{S} 1$ at varying stimulus intervals 2-7 hours after injection are illustrated in fig 1 . Recordings from 2-8 ms were obtained in five animals and $10-1000 \mathrm{~ms}$ in seven animals. Nerve fibres had generally recovered from a refractory state after 2-4 ms. Prolonged supernormality was present from 4-400 ms and had disappeared by 600 ms. Although there was some overlap in the degree of supernormality when at its height $(4-10 \mathrm{~ms})$ the mean magnitude of supernormality in the pyrethroid treated animals was greater than in the controls. The difference between the two groups was most evident from $30-400 \mathrm{~ms}$ when the subnormal period in the control animal was replaced by prolonged supernormality in those treated with deltamethrin. The values at different time intervals for control and pyrethroid treated animals were compared using Student's $t$ test. The differences were significant at $\mathrm{p}<0.001$ at all time intervals from 8-200 ms inclusive, and at $0.01>p>0.001$ at $6 \mathrm{~ms}$ and $400 \mathrm{~ms}$.

Five animals were re-examined 24-28 hours after injection and the mean values are illustrated in fig 3 . Using Students $t$ test the difference between the control and the treated animals reached significance at some stimulus intervals between $4 \mathrm{~ms}$ and $600 \mathrm{~ms}$ but not at others. The number of observations, however, was small. In order to increase the numbers for statistical comparison the results 24 hours after injection were paired with those of the same five animals prior to injection. The results for stimulus intervals from $2-10 \mathrm{~ms}$ were grouped together as were those for the intervals from $20-200 \mathrm{~ms}$. The

(closed circles). 


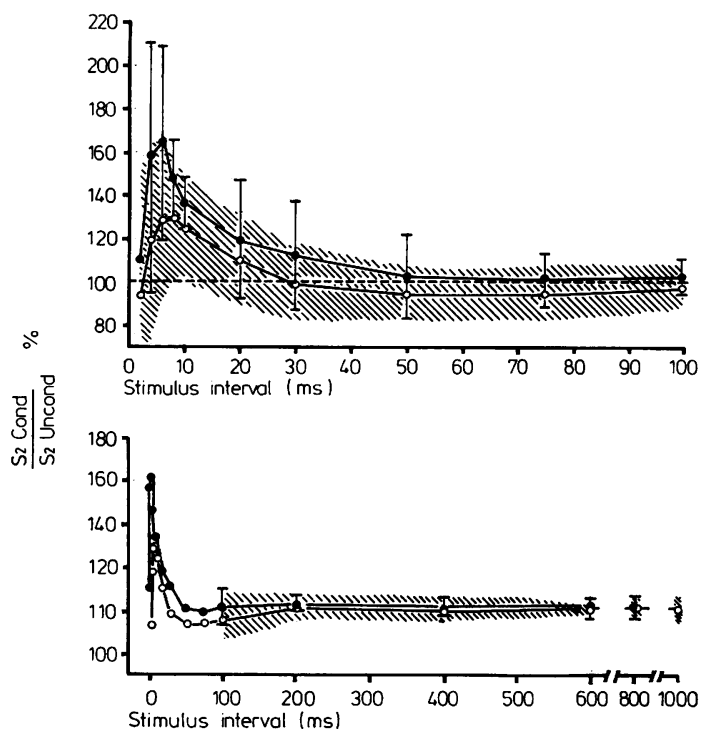

Fig 3 Control animals (open circles) and deltamethrin treated animals 24-28 hours after injection (closed circles). Stippled area denotes $\pm 2 S D$ from the mean in controls; vertical lines $\pm 2 S D$ from the mean in deltamethrin treated animals.

difference in the paired results for the $20-200 \mathrm{~ms}$ groups was significant at $\mathrm{p}<0.001$.

In further experiments the effects of incremental changes in the intensity of $\mathbf{S} 2$ on supernormality were studied, at stimulus intervals of $8 \mathrm{~ms}$ and $50 \mathrm{~ms}$. Typical results for an animal before and 3 hours after pyrethroid administration are shown in fig 4 . Because the electrodes were removed and then reapplied between the two examinations the stimulus intensity necessary to produce a maximal response and the amplitude of this response itself were different. Values have therefore been expressed as a percentage of the maximal values.

In the control recording at $8 \mathrm{~ms}$, supernormality was present at all submaximal S2 stimulus intensities and was manifest as both a reduction in the stimulus intensity necessary to produce a response of any fixed amplitude and as an increase in the amplitude of the $\mathbf{S} 2$ response when preceded by a conditioning stimulus. The administration of deltamethrin resulted in an enhancement of these effects. At $50 \mathrm{~ms}$ supernormality was clearly seen at most stimulus intensities in the treated animals.

From fig 4 it is apparent that when supernormality is expressed as a percentage change in amplitude, it will be greater at low S2 intensities and smaller at high $\mathbf{S} 2$ intensities. It was for this reason that a constant $\mathrm{S} 2: \mathrm{S} 1$ ratio (of approximately $30 \%$ ) was

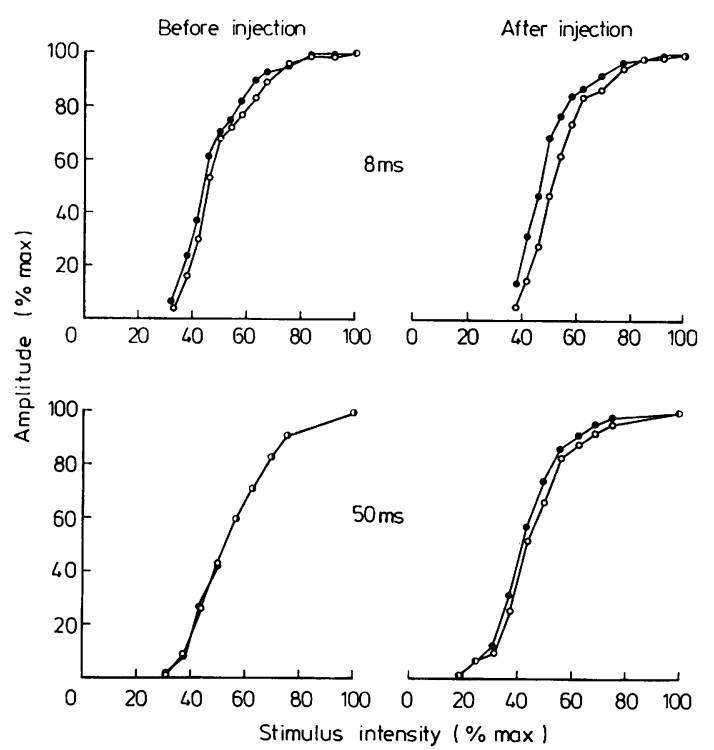

Fig 4 Effect of increasing stimulus intensity on the amplitude of response at $8 \mathrm{~ms}$ and $50 \mathrm{~ms}$, before and 3 hours following deltamethrin. Open circles unconditioned stimulus; closed circles conditioned stimulus.

chosen. Although variations in the $\mathbf{S} 2: \mathbf{S} 1$ ratio may influence the magnitude of the supernormal response, the duration of supernormality was largely unaffected by such variations and did not persist beyond $30 \mathrm{~ms}$.

\section{Discussion}

The refractory periods, supernormal period and subnormal period are the sequential changes in nerve excitability that follow propogation of a nerve impulse and are a normal physiological phenomenon in myelinated and unmyelinated nerve fibres of both the central and peripheral nervous systems of vertebrates. ${ }^{8}$ The supernormal period follows immediately upon the absolute and relative refractory periods and is demonstrable as a decrease in the excitability threshold, as an increase in the amplitude of a submaximal response in the second of two stimuli. In human median nerve this phase lasts up to 20-25 ms after the stimulus 914 and this is similar to the duration in other mammalian peripheral nerves. ${ }^{15} \mathrm{~A}$ period of subnormality excitability is often subsequently seen which may extend up to $100-200 \mathrm{~ms}$ or longer before nerve excitability returns to its resting state.

This sequence of excitability changes was confirmed in our experiments on control animals. In the sensory nerve fibres of the rat tail we found that 
a supernormal phase of excitability commenced approximately 2-4 ms after a stimulus and persisted for up to $30 \mathrm{~ms}$. A period of subnormal excitability then followed in most animals which persisted to 100-200 ms.

When these animals were treated with deltamethrin a striking augmentation of the supernormal phase occurred. This was evident not only in the magnitude of the supernormal response but was most striking as a prolongation of supernormality. The subnormal phase of excitability of the control animals was thus uniformly replaced by a markedly prolonged supernormal phase that extended up to $200-400 \mathrm{~ms}$ following a single stimulus.

Deltamethrin prolongs the inward sodium current across the nerve membrane by prolonging the duration of opening of a proportion of sodium channels and it is this effect which is likely to account for the prolonged period of supernormal excitability. Similar changes in sodium channel kinetics may be induced by the veratrum alkaloids. Veratridine, for example, is also known to prolong the sodium current across the nerve membrane ${ }^{16}$ and this results in both a prolonged negative after potential and a prolonged period of supernormal excitability. ${ }^{17}$

Although the effects on sodium channels appear to be a property common to all pyrethroids the duration differs with different compounds. Vijverberg and Van den Bercken ${ }^{5}$ suggest that gross prolongation of the sodium tail current in voltage clamp experiments may be brought about by those pyrethroids that contain an $a$-cyano group such as deltamethrin and cypermethrin. The sodium tail current produced by allethrin, which does not have an $\alpha$-cyano group, is much shorter and decays within $100 \mathrm{~ms}$. Thus a striking prolongation of supernormality such as that produced by deltamethrin would not be expected to occur with all pyrethroids.

The abnormal involuntary movements that were induced by the injection of deltamethrin were similar to those described by other workers ${ }^{18}$ and the presence of these movements precluded the study of excitability changes earlier than 2-7 hours after injection. The electrophysiological abnormalities that we observed were thus recorded at a time when the clinical signs of central nervous system intoxication had begun to resolve. 24-28 hours after injection the excitability changes induced by deltamethrin had begun to approach their control values but a minor effect was still present. The difference from control animals was most evident at longer stimulus intervals $(20-200 \mathrm{~ms})$. The difference was less marked at shorter intervals, probably because of a greater variability of the response.

Following the intravenous injection of deltamethrin in the rat, peak levels of radiolabelled fractions of the molecule are reached in cerebral tissue within minutes and have fallen within 2 hours. It is noteworthy that in the sciatic nerve radioactivity increases for up to 2 hours and significant amounts persist for as long as 8 hours after injection. ${ }^{19}$ Our results suggest that deltamethrin or its pharmacologically active metabolites may persist for even longer than this.

The dose of deltamethrin used in our experiments was sufficiently large to prove lethal to $29 \%$ of animals and we have yet to establish whether lesser doses are also associated with measurable excitability changes. It will also be important to determine whether cumulative effects occur during chronic exposure to lower doses.

Laboratory workers exposed to synthetic pyrethroids may develop transient facial sensory symptoms but conventional conduction studies in limb nerves have not shown any abnormality. ${ }^{20}$ The technique we have used in our studies in the rat is simple, non-invasive, readily repeatable and has been previously used in the study of human peripheral nerves. It is therefore possible that the study of excitability changes of the kind we have described in the rat might have some application to the electrophysiological evaluation of humans exposed to pyrethroid insecticides if we are able to detect changes in the animals following lower doses or during chronic exposure. It may also have some relevance to detecting effects of other substances which cause changes in membrane excitability.

We are most grateful to $\mathrm{Mr}$ Tarlock Gajree for his assistance throughout the study. PJP is indebted to Group Toxicology Division, Shell International Petroleum Company Ltd for a grant which enabled him to partake in the study. We would like to thank Professor TA Sears for his most helpful discussions and advice and for his criticism of the manuscript.

\section{References}

1 Verschoyle RD, Aldridge WN. Structure-activity relationships of some pyrethroids in rats. Arch Toxicol 1980;45:325-9.

2 Wouters W, Van den Bercken J. Action of pyrethroids. Gen Pharmacol 1978;9:387-98.

${ }^{3}$ Narahashi T, Anderson NC. Mechanism of excitation block by the insecticide allethrin applied externally and internally to squid giant axon. Toxicol App Pharmacol 1967;10:529-47.

${ }^{4}$ Narahashi T. Mode of action of pyrethroids. Bull WHO $1971 ; 44: 337-45$.

${ }^{5}$ Vijverberg HPM, Van den Bercken J. Frequencydependent effects of the pyrethroid insecticide decamethrin in frog myelinated nerve fibres. Eur J Pharmacol 1979;58:501-4. 
${ }^{6}$ Van den Bercken J, Vijverberg HPM. Voltage clamp studies on the effects of allethrin and DDT on the sodium channels in frog myelinated membrane. In: Insect Neurobiology and Pesticide Action. London: Soc Chem Ind, 1980:79-85.

${ }^{7}$ Lund EL, Narahashi T. Modification of sodium channel kinetics by the insecticide tetramethrin in crayfish giant axons. Neurotoxicology 1982; in press.

${ }^{8}$ Swadlow HA, Waxman SG. Activity dependent variations in the conduction properties of central axons. In: Waxman SG (ed). Physiology and Pathophysiology of Axons. New York: Raven Press, 1978:191-202.

${ }^{9}$ Gilliat RW, Willison RG. The refractory and supernormal periods of the human median nerve. $J$ Neurol Neurosurg Psychiatry 1963;26:136-47.

${ }^{10}$ Gasser HS, Erlanger J. The ending of the axon action potential and its relation to the other events in nerve activity. Am J Physiol 1930;94:247-77.

"Graham HT. Supernormality, a modification of the recovery process in nerve. Am J Physiol 1934;110: 225-42.

${ }^{12}$ Raymond SA, Lettvin JY. After effects of activity in peripheral axons as a clue to nervous coding. In: Waxman SG, ed. Physiology and Pathophysiology of Axons. New York: Raven Press, 1978:203-25.
${ }^{13}$ Misumi J. Electrophysiological studies in vivo on peripheral nerve function and their application to peripheral neuropathy produced by organic mercury in rats. II Measurement of the maximum conduction velocity by compound action potentials in the tail nerve of healthy rats. Kumamoto Med J 1979;32: $1-14$.

${ }^{14} \mathrm{Stohr}$ M. Activity-dependent variations in threshold and conduction velocity of human sensory fibres. $J$ Neurol Sci $1981 ; 49: 47-54$

${ }^{15}$ Gasser HS, Grundfast H. Action and excitability in mammalian A fibres. Am J Physiol 1936;117:113-33.

${ }^{16}$ Ulbricht W. Voltage clamp studies of veratinised frog nodes. J Cell Comp Physiol 1965;66:91-8.

${ }^{17}$ Richards $\mathrm{CH}$, Gasser HS. After potentials and recovery curve of C fibres. Am J Physiol 1935;113:108-9.

${ }^{18}$ Ray DE, Cremer JE. The action of decamethrin (a synthetic pyrethroid) on the rat. Pestic Biochem Physiol 1935;10:333-40.

${ }^{19}$ Gray AJ, Rickard J. Distribution in rats after intravenous injection of a toxic dose of ${ }^{14} \mathrm{C}$-acid, -alcohol or -cyno labelled deltamethrin. Pestic Biochem Physiol 1981;16:79-85.

${ }^{20}$ Le Quesne PM, Maxwell IC. Transient facial sensory symptoms following exposure to synthetic pyrethroids: a clinical and electrophysiological assessment. Neurotoxicology 1980;2:1-11. 